\title{
Measuring and Managing Uncertainty Through Data Fusion for Application to Aircraft Identification System
}

\author{
Peter Pong ${ }^{1}$ and Subhash Challa ${ }^{2}$ \\ ${ }^{1}$ Jacobs Australia / University of Melbourne \\ ${ }^{2}$ NICTA Victoria Research Laboratory / University of Melbourne \\ Australia
}

\section{Introduction}

Despite the use of modern Identification Friend Foe (IFF) technology, aircraft recognition remains problematic even though a great deal of research effort has already been invested in this area. In the military context, IFF identification is supposed to be initiated when the interrogator transmits a signal to the aircraft and friendly aircraft are 'supposed' to reply to the signal by transmitting an identification code to the interrogator. Hostile aircraft often become unresponsive to the interrogator because it is either does not have the appropriate transponder or is trying to avoid being identified as an unfriendly aircraft. In the civilian air transport system, the Secondary Surveillance Radar (SSR) allows the location of the civilian aircraft being transmitted (through transponder) to the Air Traffic Controller (ATC). However, in extreme incidents, such as the attacks on the World Trade Center on 11th September 2001, the SSR transponders were manually disabled, which prevented the ATC detecting flight path alternation. To avoid the drawback of the transponder based aircraft identification system, the technique of Non-Cooperative Target Recognition (NCTR) has become a useful technology, because it does not require the participation of friendly aircraft. The NCTR technique relies primarily on the ground based target classification technology. In a typical classification problem, the goal is to develop a classifier that is capable to discriminate targets. This technology shares a great deal of similarity with the modern Electronics Support Measures (ESM) system that often employs as a Radar Warning Receiver (RWR) for modern military aircraft self-protection. Acknowledging the number of successful classifier technologies reported in this area, the goal of this work is not to propose any new algorithm to enhance the classification technology. Instead, a novel method, based on uncertainty measures, is introduced to improve the classification function by employing a data fusion technique. Data fusion applying evidential reasoning framework is a well established technique to fuse diverse sources of information. A number of fusion methods within this formalism were introduced including Dempster-Shafer Theory (DST) Fusion, Dezert Samarandche Fusion (DSmT), and Smets' Transferable Belief Model (TBM) based fusion. However, the impact of fusion on the level of uncertainty within these techniques was not studied in detail. While the use of Shannon entropy with the Bayesian fusion is well understood, the measures of uncertainty within the Dempster-Shafer formalism is not widely regarded. In this paper, an uncertainty based technique is proposed to quantify the evolution of DST fusion. This technique is then 
utilised to determine the optimal combination of sensor information to achieve the least uncertainty in the context of the aircraft identification problem using sensors operating the NCTR technique.

\section{Background}

Information fusion is often used as a data-processing technique to integrate uncertain information from multiple sensors. Information often contains uncertainties, which are usually related to physical constrains, detection algorithms and the transmitting channel of the sensors. Whilst the intuitive approaches, such as Dempster-Shafer Fusion (Shafer, 1976), Dezert Samarandche Fusion (DSmT)(Dezert \& Smarandache, 2006) and Smets' Transferable Belief Model (TBM) (B.Ristic \& P.Smets, 2005) aggregate all available information, these approaches do not always guarantee optimum results. Acknowledging that these techniques have associated measurement costs, the essence is to derive a fusion technique to minimise global uncertainties.

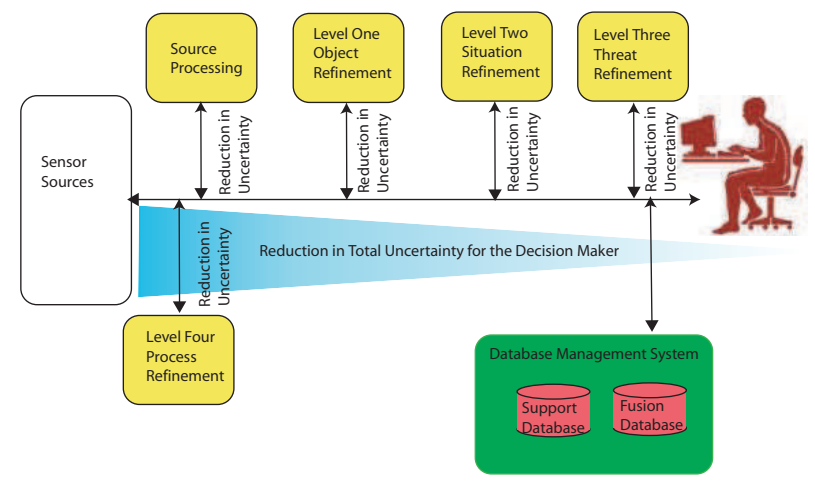

Fig. 1. JDL Model and Uncertainty

In the aerospace community, there is an increasing trend to automate decision processes based on information fusion techniques. As an example, fighter pilots may rely on various forms of data fusion models to assist in assessing the current situations, when uncertain information co-exists at all levels of fusion. Considering the many data fusion models, the Joint Defence Laboratory (JDL) model (Hall \& Llinas, 2001) is one the most commonly referred frameworks, which consists of Level 1 Object Assessment, Level 2 Situation Assessment, Level 3 Impact Assessment and Level 4 Process Refinement. The decision maker is supposed to treat the JDL model at 4 independent levels of functions, however, each level of fusion often includes unavoidable uncertainties. That means any aircraft identification system employing real-time situation analysis technology is required to manage uncertainty in the most effective manner. The techniques based on statistical models employed in aircraft tracking were widely acknowledged, but the methods based on uncertainty measures for target identification are not well understood in the aviation community. In recognition of this deficiency, this paper explores a novel aircraft identification technique by leveraging a new uncertainty based fusion concept.

The new concept introduced in this work explores a number of uncertainty measures under the reasoning framework and attempts to introduce a methodology to manage uncertainty variation under the DST based fusion. An example derived from an Aircraft Identification (AI) 


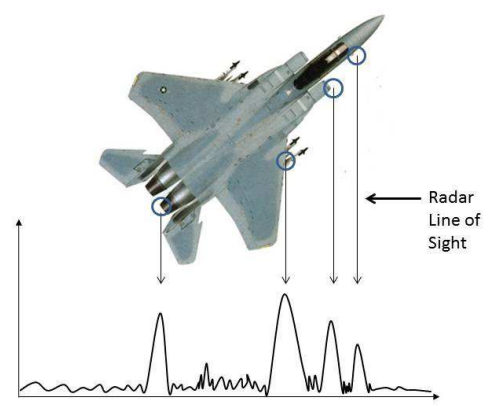

Fig. 2. Example of a radar range profile of a fighter aircraft

system is employed to demonstrate the characteristics of uncertainty variation. In terms of target tracking, significant advancements have been made in the past two decades to improve tracking technology by employing sophisticated data fusion techniques. Some of the earlier works went even further by incorporating Target Identification information, such as IFF data, to improve the overall track quality (Leung \& Wu, 2000), (Carson \& Peters, 26-30 Oct 1997), (Bastiere, 1997), and (Perlovsky \& Schoendorf, 1995). When legitimate statistical information is presented, the techniques employed by tracking and identification using IFF information are relatively mature. However, when conflicting information is presented to the NCTR system, most techniques employed today may find it difficult to discriminate the contradicting information. In this work, we propose a technique based on uncertainty measures to resolve this problem. The employment of uncertainty in recent aviation research was reported in areas, such as air traffic control (Porretta \& Ochieng, 2010), navigation (Deng \& Liu, 2011) and airport surface movement management (Schuster \& Ochieng, 2011), however, all these works essentially model uncertainty based on the target statistical characteristics, such as model based classified illustrated in Figure 2. Instead of treating uncertainty implicitly using their statistical values, the concept proposed in this work treats uncertainty measures directly as input parameters. In this way, we could explicitly quantify the fusion performance to make the best target identification.

\section{Sensor selection and decision making}

Information fusion is often perceived to produce improved decision. This assumption is generally true when sensor availability is limited, however, one has to question whether fusing all available data guarantee synergy. The focus of this work is on the reduction of uncertainties by expressing the relevant uncertainties in the reasoning system and utilise these measures to achieve the best information fusion strategy. In order to develop an uncertainty based information fusion in the aircraft identification context, the authors argue that the best fusion decision can only be observed when (i) the information fusion could provide the least ambiguous choice, (ii) the result produced by the fusion system induces the least vague answer under the reasoning framework, and (iii) the final recommendation provided by the fusion system has the fewest uncertainties. These three axioms underlying this paper are used 
to define the best fusion configuration. It is apparent that the goal of uncertainty based fusion is to choose the result with the least uncertainty. A fusion process based on uncertainties has the potential to lead to a biased result. However, it is difficult to neglect a decision based on information fusion when it is the least uncertain, least ambiguous and the most defined answer when compared with other potential solutions.

Figure 3 depicts an illustrative example where an aircraft identification scenario is considered. Assuming a model based classifier is employed to identify three kinds of aircraft types Dual engines aircraft (D), Quadruple engines aircraft $(\mathrm{Q})$ and Helicopter $(\mathrm{H})$. Also assuming that the sensors produced an "unknown" state in the form of $\{D, Q, H\}$, where the decision of the aircraft type is not possible to be classified. Three sensors are utilised in this example to simplify the demonstration, where a classification value based on Basic Probability Assignment (BPA) are given to each of the classification reports with details also summarised in Figure 3.

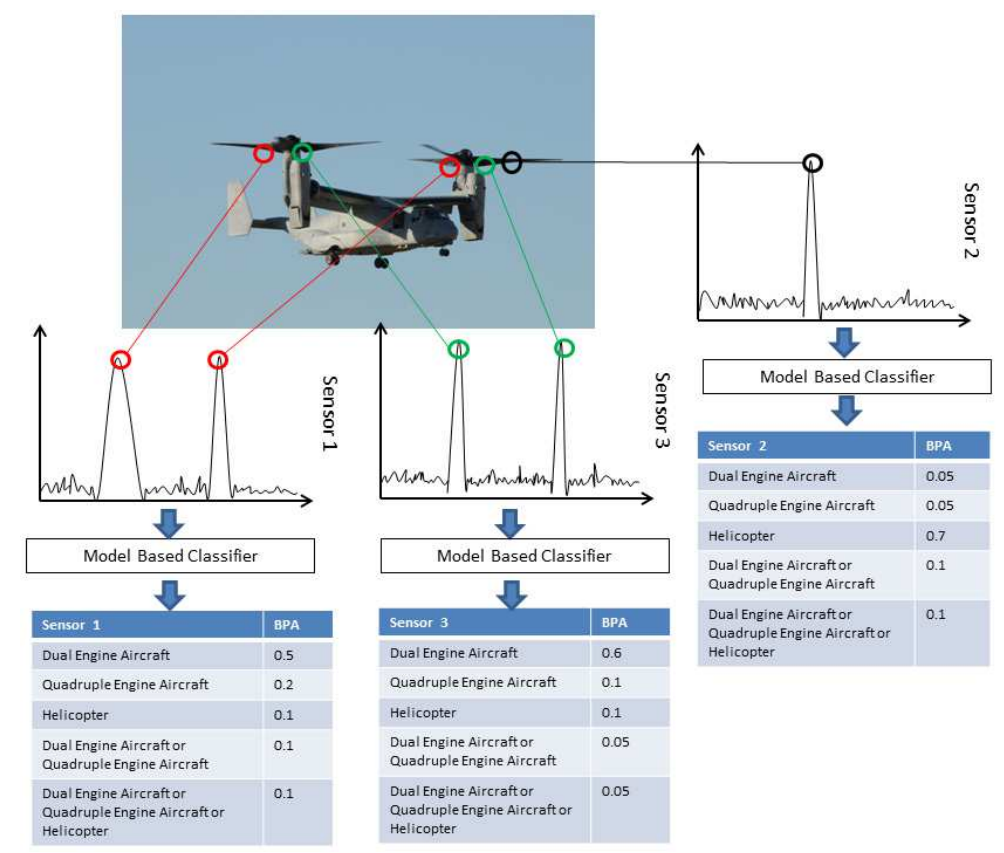

Fig. 3. Multi-sensor aircraft classification

If the identification process performed by each sensor is independent, information provided by Sensor 2 is clearly contradicting with Sensor 1 and Sensor 3. The errors can be induced by the incorrect scatter angle, or simply estimated by an inaccurate model. Based on the axioms discussed, it is observed that fusing Sensor 1 and Sensor 2, or Sensor 2 and Sensor 3 under DST (which be discussed in the next section) will not produce a pronounced result to identify the aircraft type. The result of the fusion is illustrated in Table 1, where only the combination of Sensor 1 and Sensor 3 could provide an unambiguous fusion result. This example highlights the criticality of uncertainty measures in relation to the standard DST fusion process. Section 5 and Section 6 of this paper provide an empirical uncertainty measures analysis in the 


\begin{tabular}{|c|c|c|c|}
\hline & Sensor 1\&2 & Sensor 1\&3 & Sensors 2\&3 \\
\hline $\mathrm{D}, \mathrm{Q}, \mathrm{H}$ & 0.026 & 0.022 & 0.0356 \\
\hline $\mathrm{D}, \mathrm{Q}$ & 0.0779 & 0.037 & 0.0595 \\
\hline $\mathrm{H}$ & 0.3506 & 0.7704 & 0.3810 \\
\hline $\mathrm{Q}$ & 0.1558 & 0.1185 & 0.0833 \\
\hline $\mathrm{D}$ & 0.3896 & 0.0519 & 0.4405 \\
\hline
\end{tabular}

Table 1. Sensor fusion example with contradicted information

reasoning framework, and provides an insight into how this method can be applied in an aircraft identification capability.

\section{Evidential reasoning framework}

The notion of Basic Probability Assignment (BPA) (Shafer, 1976) is defined with respect to a finite universe of propositions or frame of discernment, $\Omega$. The sum of the probabilities assigned to all subsets of $\Omega$ and all propositions which support $\Omega$ must be in unity, as such $B P A$ is a function from the set of subsets, $2^{\Omega}$, of $\Omega$ to the unit interval $[0,1]$. In accordance with the convention proposed by Shafer (Shafer, 1976):

$$
m(\varnothing)=0
$$

and

$$
\sum_{A \subseteq \Omega} m(A)=1
$$

The subset $A$ of $\Omega$ such that $m(A)>0$ is called a focal element of $m$, and $\varnothing$ is the empty set. Whilst the summation of BPA must be unity, it is not manditory for the BPA of a proposition $A$ and its negation $\bar{A}$ sum to unity.

\subsection{Belief and plausibility measures}

The idea of linking belief with evidential measures was first discussed by Shafer, and the idea of Belief function in reference to the BPA is defined as,

Definition 1. Bel: $2^{\Omega} \rightarrow[0,1]$ is a belieffunction over $\Omega$ if it satisfies:

- $\operatorname{Bel}(\varnothing)=0$

- $\operatorname{Bel}(\Omega)=1$

- for every integer $n>0$ and collection of subsets $A_{1}, \ldots ., A_{n}$ of $\Omega$

$$
\operatorname{Bel}\left(A_{1} \cup \ldots \cup A_{n}\right) \geq \sum_{i} \operatorname{Bel}\left(A_{i}\right)-\sum_{i<j} \operatorname{Bel}\left(A_{i} \cap A_{j}\right)+\ldots+(-1)^{n+1} \operatorname{Bel}\left(A_{1} \cap \ldots \cap A_{n}\right)
$$

BPA gives a measure of support that is assigned exactly to the focal elements of a given frame of discernment. In order to aggregate the total belief in a subset $A$, the extent to which all the available evidence supports $A$, one needs to sum together the BPAs of all the subsets of A for a belief measurement.

$$
\operatorname{Bel}(A)=\sum_{B \subseteq A} m(B) \quad \forall A \subseteq \Omega
$$


The remaining evidence may not necessarily support the negation $\bar{A}$. In fact some of them may be assigned to propositions which are not disjointed from $A$, and hence, could be plausibly transferred directly to $A$ for further information. Shafer called this the plausibility of A:

$$
\operatorname{Pl}(A)=\sum_{B \cap A \neq \varnothing} m(B) \quad \forall A \subseteq \Omega
$$

\subsection{Dempster-Shafer fusion under an iterative process}

Dempster's rule of combination forms a new body of evidence with which the focal elements are all non-empty intersections $X \cap Y$. Given any $S \subseteq U$ there are many pairs $X, Y \subseteq U$ such that $X \cap Y=S$ and so the total weight of agreement assignable to the focal subset $X \cap Y$ is $\sum_{X \cap Y=S} m(X) m^{\prime}(Y)$. Once normalising the agreement with the "non-conflicting values" $(1-K)$, Dempster's rule of combination for imprecise evidence becomes,

$$
\left(m * m^{\prime}\right)(S)=\frac{1}{1-K} \sum_{X \cap Y=S} m(X) m^{\prime}(Y)
$$

for all $\varnothing \neq S \subseteq U$. The conflict between two bodies of evidence $m, m^{\prime}$ is the total weight of contradiction between the events of $m$ and the events of $m^{\prime}$ :

$$
K\left(m, m^{\prime}\right)=\sum_{X \cap Y=\varnothing} m(X) m^{\prime}(Y)
$$

The quantity $1-K$ is the cumulative degree to which the two bodies of evidence do not contradict with each other and is called the agreement between $m$ and $m^{\prime}$. In general evidential theory, Dampster-Shafer rules, belief functions, plausibility functions and BPA forms a suite of significant tools to construct probabilities through carefully modelled evidence. Through this combination process, two new measurement values - non-specificity and conflict, are also generated as a by-product. An empirical analysis is presented in Section 5 in conjunction with the theory of Aggregated Uncertainty (AU) and the recently proposed generalised Total Uncertainty (TU) measures.

\section{Uncertainty measures within the evidential reasoning framework}

While the classical uncertainties are often measured by the Hartley and Shannon functions, the two functions are tailored for different purposes. In order to cater for both uncertainties, evidential based uncertainty measures are adopted. Two types of classical evidential based uncertainties - non-specificity and conflict - are often measured as part of the DST fusion (Harmanec, 1996). In this section, an overview is introduced to the concept of Hartley Uncertainty measures, Aggregrated Uncertainty (AU) measures and Total Uncertainty (TU) measures which was proposed by Klir (Klir, 2006). This analysis covers the context of the DST fusion system and their subsequent implication. A practical example based on aircraft identification applying uncertainty measures as sensor discrimination matrices is discussed in Section 7 to verify our observations.

\subsection{Hartley uncertainty}

The technique of uncertainty measures was first addressed by Shannon. Under his proposal, the way to quantify uncertainty measures expressed by a probability distribution function $p$ 
on a singleton set is in the form of,

$$
-c \sum p(x) \log _{b} p(x)
$$

where $b$ and $c$ are positive constants, and $b \neq 1$. While this technique is useful to apply in sensor management system operating under the probabilistic framework, it cannot be used under a finite set condition. An alternative is to employ the legacy Hartley measures (Hartley, n.d.), where it seems to be the only meaningful way to measure uncertainty in the form of,

$$
c \log _{b} \sum_{x \in \Omega} r_{A}(x)
$$

or alternatively

$$
c \log _{b}|A|
$$

where $A$ is a finite set and $|A|$ is the cardinality of the finite set. $b$ and $c$ are positive constants, and $b \neq 1$. When uncertainty is measured in bits, $c \log _{b} 2=1$. Harley uncertainty measures, $H$, defined for any basic possibility functions, $r_{A}$,

$$
H\left(r_{A}\right)=\log _{2}|A|
$$

On closer examination of (10), $H\left(r_{A}\right)$ is a measure directly related to the specificity of a finite set. In other words, the larger the size of a set, the less specific the measurement becomes. This type of measures was defined as non-specificity by Klir (Klir, 2006). In the reasoning framework, Hartley Measures are usually treated as a weighted average of all the focal subsets in the form of BPA function (Klir, 2006). The concept of generalised Harley measures in the context of DST framework is thus defined by the function,

$$
G H(m)=\sum_{A \in \Omega} m(A) \log _{2}|A|
$$

where $\Omega$ is the superset of the focal elements.

\subsection{Aggregated uncertainty measures}

Suppose the goal of information fusion is to reduce global uncertainties, Harmanec (Harmanec, 1996) was the first to explore the concept of uncertainty measures in the DST framework. The idea of $A U$ uncertainty measures was proposed as the optimum uncertainty measures technique under the DST domain, because it is the only way to incorporate the value of non-specificity and conflict simultaneously, which often coexist in the DST framework.

Definition 2. The measure of the Aggregated Uncertainty contained in Bel, denoted as $A U(B e l)$, is defined by

$$
A U(B e l)=\max \left\{-\sum_{x \in \Omega} p_{x} \log _{2} p_{x}\right\}
$$

where the maximum is taken over all $\left\{p_{x}\right\}_{x \in \Omega}$ such that $p_{x} \in[0,1]$ for all $x \in \Omega, \sum_{x \in \Omega} p_{x}=1$ and for all $A \subseteq \Omega, \operatorname{Bel}(A) \leq \sum_{x \in A} p_{x}$.

Although the $A U$ technique is not an efficient algorithm, it does satisfy all the properties defined as uncertainty measures (Harmanec, 1996), and specifically, the subadditivity/additivity characteristics. 


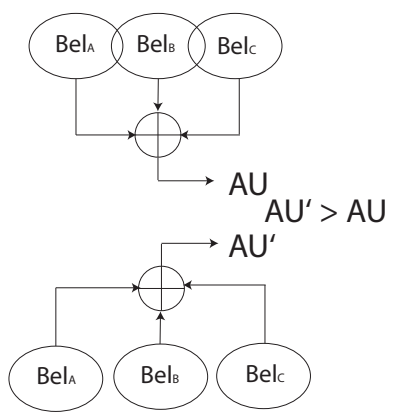

Fig. 4. Additivity and Subadditivity

Subadditivity. If $\mathrm{Bel}$ is an arbitrary joint belief function on $X \times Y$ and the associated marginal belief functions are $\mathrm{Bel}_{X}$ and $B e l_{Y}$, then

$$
A U(B e l) \leq A U\left(\mathrm{Bel}_{X}\right)+A U\left(\mathrm{Bel}_{Y}\right)
$$

Additivity. If Bel is a joint belief function on $X \times Y$, and the marginal belief functions Bel $_{X}$ and $\mathrm{Bel}_{Y}$ are noninteractive, then

$$
A U(B e l)=A U\left(B e l_{X}\right)+A U\left(\text { Bel }_{Y}\right)
$$

The property of additivity/subadditivity of AU call forth the assumption that uncertainties could be reduced if sensors share common interaction prior the information fusion process occurring. Assuming sensor dependency exists among $\mathrm{Bel}_{A}, \mathrm{Bel}_{B}$ and $B e l_{C}$, the characteristics of the resultant uncertainty under an evidential fusion system is illustrated pictorially in Figure 4. The algorithm to compute AU uncertainty was originated by Harmanec (Harmanec, 1996). Under the proposed algorithm, the input is treated in the form of a frame of discernment $X$, with a belief function $\mathrm{Bel}$ on $\mathrm{X}$. This algorithm's computation completes once a finite number of steps have been taken and the output is the correct value of the function $A U(\mathrm{Bel})$, since $\left\{p_{x}\right\}_{x \in X}$ maximises the Shannon entropy within the constraints induced by Bel.

\subsection{Total uncertainty measures}

The concept of generalised Total Uncertainty (TU) was proposed by Klir (Klir \& Smith, 2001) not long after the introduction of AU uncertainty. This measure is defined as a combination of $A U$ uncertainty and Generalised Hartley Measures,

$$
T U=\langle G H, G S\rangle
$$

where GH represent the Generalised Hartley measures which was discussed in (11). The factor GS is called Generalised Shannon measurement (Klir, 2006), which is the conflicts measurement with the consideration of evident specificity. In other words, it is $G S=$ $A U-G H$, the Aggregated Uncertainty with the reduction of specificity consideration. One advantage of the disaggregated TU, in comparison with $\mathrm{AU}$, is that it expresses amounts of both types of uncertainty (non-specificity and conflict) explicitly, and consequently, it is highly sensitive to changes in evidence. These new features of uncertainty measures allow one to work with any set of recognised and well-developed theories of uncertainty as a whole, which are commonly seen in any evidential based fusion problem. 


\begin{tabular}{|c|c|c|c|}
\hline Classification & 1 Sensor & 3 Sensors & 7 Sensors \\
\hline A & 0.22 & 0.3485 & 0.4125 \\
\hline B & 0.25 & 0.3309 & 0.3525 \\
\hline C & 0.26 & 0.2845 & 0.2343 \\
\hline D & 0,00 & 0.0015 & 0.0001 \\
\hline A,B & 0.07 & 0.0163 & 0.0004 \\
\hline A,C & 0.03 & 0.005 & 0.0001 \\
\hline A,D & 0.03 & 0.005 & 0.0001 \\
\hline B,C & 0.015 & 0.0022 & 0.0000 \\
\hline B,D & 0.005 & 0.0007 & 0.0000 \\
\hline C,D & 0.01 & 0.0014 & 0.0000 \\
\hline A,B,C,D & 0.1 & 0.0042 & 0.0000 \\
\hline
\end{tabular}

Table 2. Classification Results with DST Fusion

\section{Analysis of uncertainty measures under the Dempster Shafer fusion framework}

To appreciate the impact of uncertainty variation, an example with a set of arbitrary data is illustrated in Table 2. The data set is exactly the same measurement values, such that an iterative DST fusion can be performed. The results in Table 2 confirmed that sensor information can be refined and appears to have a reduction of ambiguity under an iterative DST fusion process. However, the merit of these results cannot be examined further, unless an acceptable matrices is used to quantify the fusion. To address this point, the results illustrated in Figure 5 a demonstrate how AU uncertainty reduction could quantify the DST fusion process. Whilst the AU uncertainty measure are a useful index to quantify the DST fusion process, it is suggested to be insensitive to small change in evidences (Klir, 2006). Acknowledging the inherited issues with the AU uncertainty measures, this work also examines the concept of employing Total Uncertainty Map (TUM) to evaluate a standard DST Fusion process. Considering TU is an amalgamation of GH and GS, the uncertainty variation becomes significant if it is illustrated in two dimensional space. Figure $5 \mathrm{~b}$ is an illustration of how a TUM can be used to visualise the recursive DST fusion. To assist the interpretation, the results of GS / GH are also provided in Figure 5a to enhance the illustration. In this case, GS and $G H$ are treated as an unified parameters with the variation under the DST fusion process observed. Due to the equivalent sensor input for the DST fusion, the weighted average of

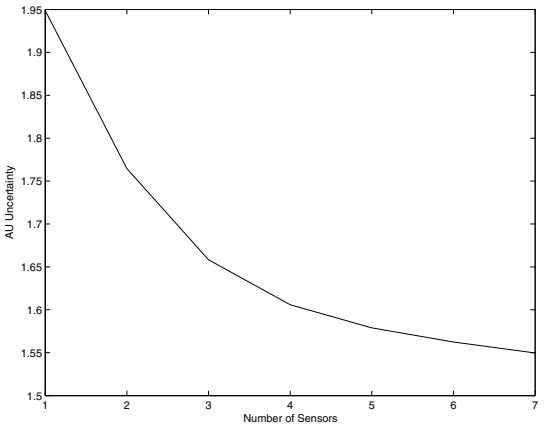

(a) AU Uncertainty Variation Under the DS Fusion

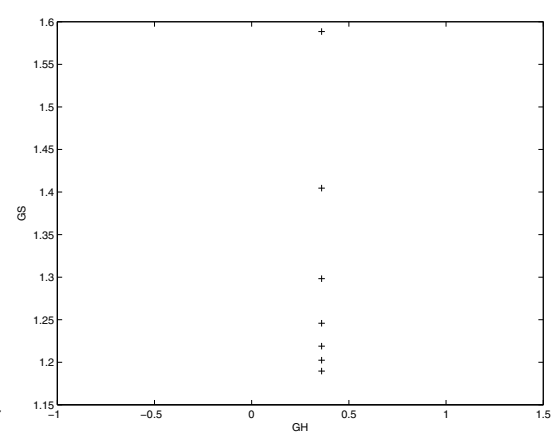

(b) TU Map Variation Under the DS Fusion

Fig. 5. Uncertainty Variation 


\begin{tabular}{|c|c|c|c|}
\hline Sensor 1 & Sensor 2 & Sensor 3 & Sensor 4 \\
\hline$\{\mathrm{A}\}=0.26$ & $\{\mathrm{~B}\}=0.2$ & $\{\mathrm{~B}\}=0.1$ & $\{\mathrm{~A}\}=0.05$ \\
$\{\mathrm{~B}\}=0.26$ & $\{\mathrm{~A}, \mathrm{~B}\}=0.1$ & $\{\mathrm{C}\}=0.1$ & $\{\mathrm{~B}\}=0.05$ \\
$\{\mathrm{C}\}=0.26$ & $\{\mathrm{~A}, \mathrm{C}\}=0.1$ & $\{\mathrm{~A}, \mathrm{~B}\}=0.16$ & $\{\mathrm{D}\}=0.2$ \\
$\{\mathrm{~A}, \mathrm{~B}\}=0.07$ & $\{\mathrm{~A}, \mathrm{~B}, \mathrm{C}\}=0.1$ & $\{\mathrm{~B}, \mathrm{C}\}=0.14$ & $\{\mathrm{~A}, \mathrm{~B}\}=0.11$ \\
$\{\mathrm{~A}, \mathrm{C}\}=0.01$ & $\{\mathrm{~A}, \mathrm{C}, \mathrm{D}\}=0.1$ & $\{\mathrm{~B}, \mathrm{D}\}=0.05$ & $\{\mathrm{~A}, \mathrm{C}\}=0.03$ \\
$\{\mathrm{~A}, \mathrm{D}\}=0.01$ & $\{\mathrm{~B}, \mathrm{C}, \mathrm{D}\}=0.3$ & $\{\mathrm{~A}, \mathrm{C}\}=0.1$ & $\{\mathrm{~A}, \mathrm{D}\}=0.03$ \\
$\{\mathrm{~B}, \mathrm{C}\}=0.01$ & & $\{\mathrm{~A}, \mathrm{~B}, \mathrm{C}\}=0.2$ & $\{\mathrm{C}, \mathrm{D}\}=0.03$ \\
$\{\mathrm{~B}, \mathrm{D}\}=0.01$ & & $\{\mathrm{~B}, \mathrm{C}, \mathrm{D}\}=0.15$ & $\{\mathrm{~B}, \mathrm{C}, \mathrm{D}\}=0.3$ \\
$\{\mathrm{C}, \mathrm{D}\}=0.01$ & & & $\{\mathrm{~A}, \mathrm{~B}, \mathrm{C}, \mathrm{D}\}=0.2$ \\
$\{\mathrm{~A}, \mathrm{~B}, \mathrm{C}, \mathrm{D}\}=0.1$ & & & \\
\hline
\end{tabular}

Table 3. Random Sensor Input

each focal subset are virtually unchanged, which is why the GH values displayed in Figure $5 \mathrm{~b}$ remain constant throughout the iterative DST fusion process. Further observation shows, however, that other uncertainty in the form of conflicts are gradually reduced as part of the DST fusion process. To further explore the characteristics of uncertainty variation, four arbitrary sensor data sets are outlined in Table 3. The TU uncertainty is displayed in Figure $6 \mathrm{~b}$. These results are further broken down into four levels and each level represents the number of sensors fused by the DST fusion. Based on the sample results, it is difficult to provide a consolidated uncertainty variation within the DST fusion framework. However, a potential optimisation solution exists when the fusion goal is to present the most specific and least conflicted information to the decision maker. This concept will be covered in Section 7 by leveraging a NCTR based AI example.

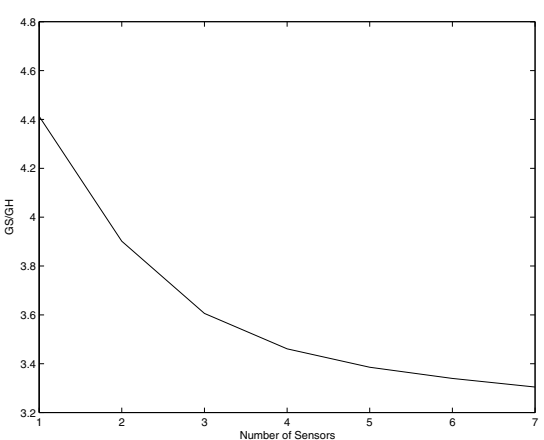

(a) GS/GH Variation Under the DS Fusion

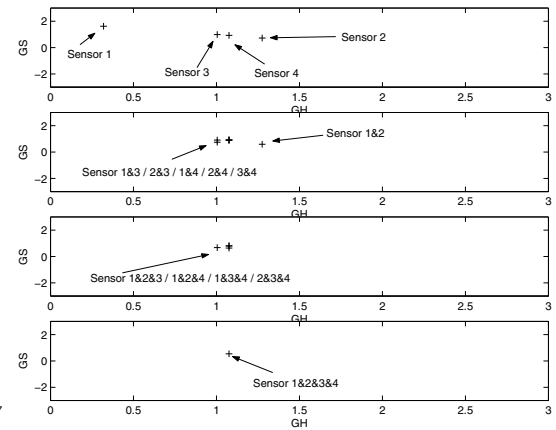

(b) TU Map Under the DS Fusion with Random Sensors Input Data

Fig. 6. Extended Uncertainty Variation Modelling

\section{NCTR based Aircraft Identification (AI)}

This case study utilises an example commonly encountered in a model based classification system. Assuming each NCTR sensor has a potential to produce feature detection of,

$$
B=\{E 0, E 1, E 2, \ldots . ., E 36\}
$$

where B is the frame of discernment of the aircraft's type attributes, and this example allows seven model based classifiers to report aircraft type identification. To reduce the 


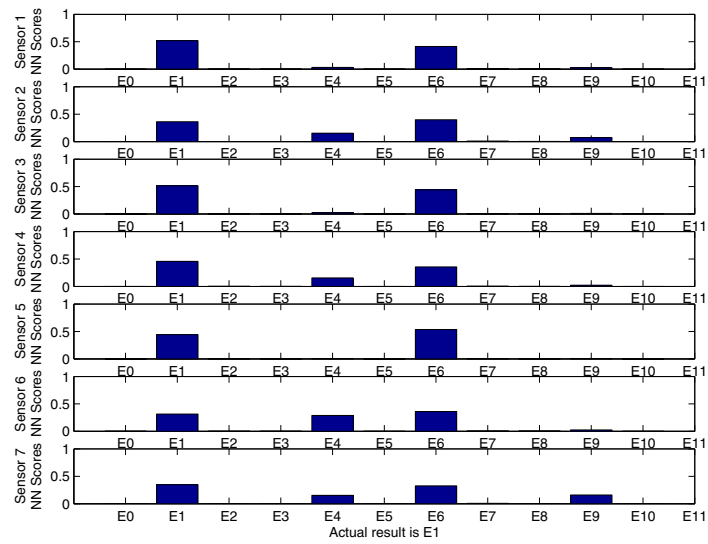

Fig. 7. Model based classifier for aircraft type detection

\begin{tabular}{|c|c|c|c|c|c|}
\hline Sensor1 & Sensor2 & Sensor3 & Sensor4 & Sensor5 & Sensor6 \\
\hline$m\left\{e_{1}\right\}=0.5188$ & $m\left\{e_{1}\right\}=0.3617$ & $m\left\{e_{1}\right\}=0.5126$ & $m\left\{e_{1}\right\}=0.4565$ & $m\left\{e_{1}\right\}=0.4414$ & $m\left\{e_{1}\right\}=0.3480$ \\
$m\left\{e_{6}\right\}=0.4124$ & $m\left\{e_{4}\right\}=0.1540$ & $m\left\{e_{6}\right\}=0.4387$ & $m\left\{e_{4}\right\}=0.1551$ & $m\left\{e_{6}\right\}=0.5342$ & $m\left\{e_{4}\right\}=0.1533$ \\
$m\{\Theta\}=0.0687$ & $m\left\{e_{6}\right\}=0.3971$ & $m\{\Theta\}=0.0487$ & $m\left\{e_{6}\right\}=0.3546$ & $m\{\Theta\}=0.0244$ & $m\left\{e_{6}\right\}=0.3254$ \\
& $m\left\{e_{9}\right\}=0.0733$ & & $m\{\Theta\}=0.0337$ & & $m\left\{e_{9}\right\}=0.1602$ \\
& $m\{\Theta\}=0.0138$ & & & & $m\{\Theta\}=0.0357$ \\
\hline
\end{tabular}

Table 4. Normalised Aircraft Detection

computational workload this example only employs 12 of the target type signature instead of the potential 37 type of targets, where the results are depicted in Figure 7 . The 12 aircraft type signatures selected for this simulation share similar characteristics, and often cause confusion to this particular NCTR platform. The remaining 25 emitter detections are not discarded, but are consolidated as detection CLUTTER. This method is similar to the strategy reported in (Yu \& Sycara, 2006), instead this case study treats all aircraft signatures as the total frame of discernment $\Theta_{E},\left\{e_{0}, e_{1}, e_{2}, e_{3}, e_{4}, e_{5}, e_{6}, e_{7}, e_{8}, e_{9}, e_{10}, e_{11}\right\}$. In terms of the simulation, each emitter signature is considered as $e_{i} \in E$, where $m\left(e_{i}\right)$ is the normalised confidence level assigned by the post threshold detection process. For instance, the normalised post-detection confidence level with Sensor 2 are $m\left\{e_{1}\right\}=0.3617, m\left\{e_{4}\right\}=0.1540, m\left\{e_{6}\right\}=0.3971$ and $m\left\{e_{9}\right\}=0.0733$. To include the non-mutually exclusive aircraft type as CLUTTER, $m\left\{\Theta_{E}\right\}=c(C L U T T E R)$, where we assign the confidence of CLUTTER to the set of all possible aircraft types. In this case, the normalised $m\left\{\Theta_{E}\right\}$ based on the pre-detection process is 0.0138 .

Upon completion with the BPA preparation, we performed a DST based fusion with a permutation space of $2^{7}$. Figure 8 shows the uncertainty in the form of AU as gradually reduced with the increment of DST fusion. However, the results become less effective when more sensors are fused together. In accordance with the discussions covered in Section 6, the authors believe the optimum approach when conducting an uncertainty based DST fusion cannot rely on one single parameter alone. Depending on the computational workload and the tolerance of conflicts, the uncertainty based fusion process ought to be determined by a TU map, where GS and GH are to be treated separately. The preliminary results based on this concept are illustrated in Figure 9. 


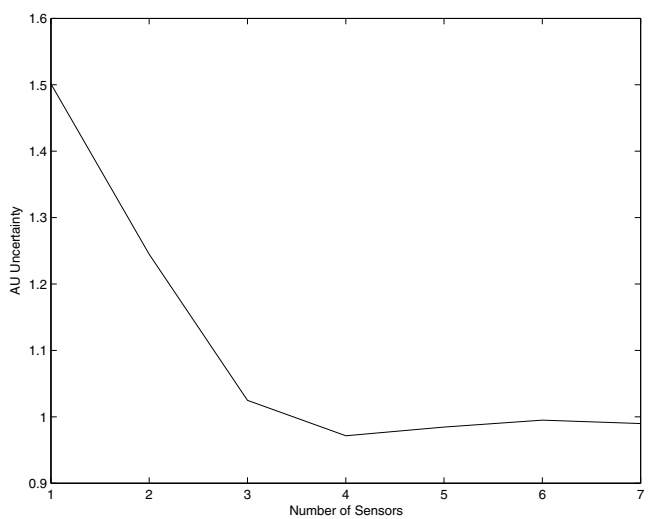

Fig. 8. AU Uncertainty Variation Under the model based classifier DST Fusion
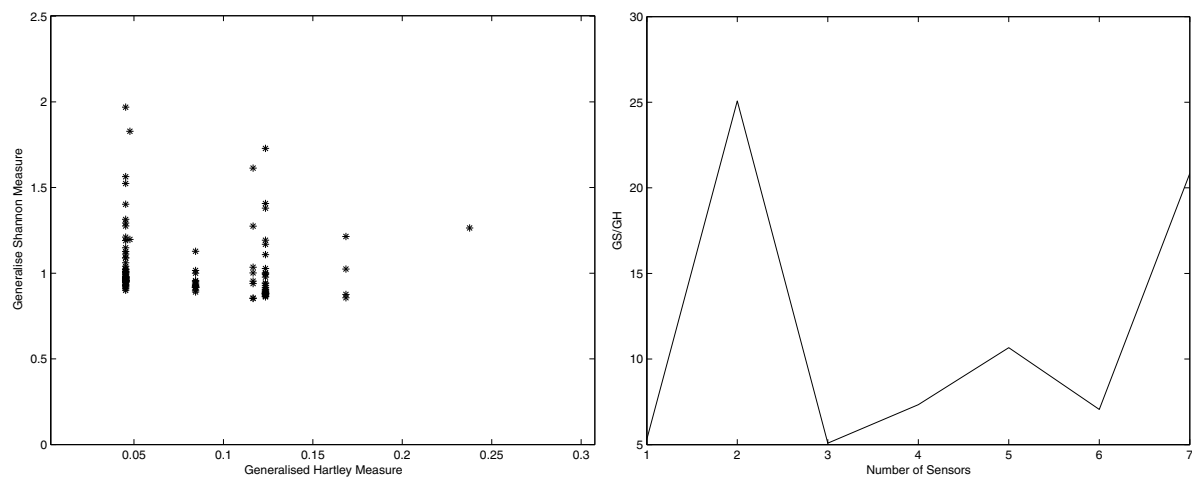

(a) TU Map Variation Under the model based (b) GS/GH Variation Under the model based classifier DST Fusion classifier DST Fusion

Fig. 9. Uncertainty Variation

Notwithstanding the treatment of uncertainty in the DST context, Figure 9a outlined a method when adopting the theory of AU uncertainty to search for the least uncertain post-fusion results. For comparison purposes, the results of GS / GH measures are also displayed in Figure $9 \mathrm{~b}$. Under such a process, the final result is to be determined by the fusion that produces the minimum AU uncertainty. In this particular example, Sensors 1, 3, 4 and 7 can be selected to participate in the fusion process. Based on the least AU uncertainty, the final BPA for the detected emitters are given below:

$$
\begin{gathered}
m\left\{\Theta_{E}\right\}=0 \\
m\left\{e_{1}\right\}=0.5604 \\
m\left\{e_{6}\right\}=0.4396
\end{gathered}
$$

With a similar approach, and adopting the GS/GH characteristics, Sensors 1, 2 and 3 are selected to join the fusion process. Based on the least GS / GH uncertainty, the final BPA for 

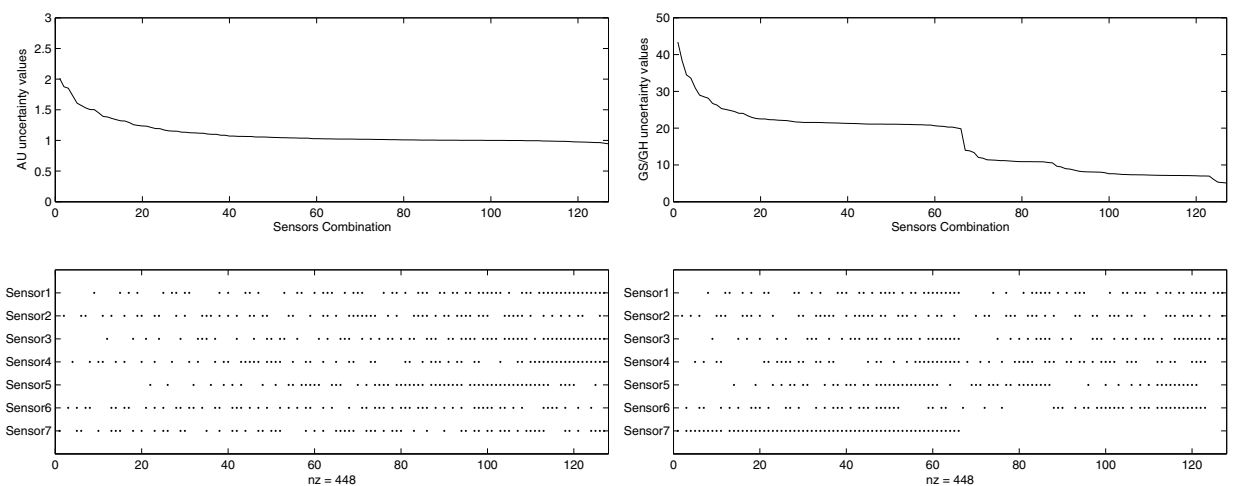

(a) AU Uncertainty measurements in conjunction (b) with sensor fusion

(b) GS/GH Uncertainty measurements in conjunction with sensor fusion

Fig. 10. Uncertainty Variation

the detected emitters are given below, which is equivalent to sensor combination with the least AU uncertainty:

$$
\begin{gathered}
m\left\{\Theta_{E}\right\}=0 \\
m\left\{e_{1}\right\}=0.5604 \\
m\left\{e_{6}\right\}=0.4396
\end{gathered}
$$

Although the final results obtained from the uncertainty based DST fusion do not yield distinct decisions, the results justify that aircraft type $e_{1}$ or aircraft type $e_{6}$ are detected.

\section{Conclusion}

This paper reviews the role of uncertainty measures in the data fusion framework within the context of evidential reasoning. An empirical analysis of the AU and TU uncertainty variations is conducted under the DST fusion framework. A preliminary method to choose sensors based on the uncertainty level is proposed. This technique is illustrated with an aircraft identification problem, when the radar range profile classifier is employed to support an identification system such as NCTR. Since the amount of reflected radar energy is different for different parts of the aircraft, inconsistency often occurs even when the same target is being observed by a number of sensors despite using the same classifier model. It is this inconsistency which makes the uncertainty based fusion technique useful in resolving aircraft identification problems. While the proposed technique can be computationally intensive, the idea underwrites a conservative result with the least measurable uncertainty. This approach essentially yields the potential to evaluate all kinds of reasoning based fusion systems. We have certainly not reached the end of our research effort yet, as the proposed concept only considers primarily the reduction of AU uncertainty. The authors recognise the benefits in further investigation of TUM in conjunction with the theory of optimisation, when a trade-off can be computed based on the classification's precision and accuracy. At the moment, our proposed concept does not take into account the sensor information based on human originated data. It is certainly an exciting future research topic, if the proposed concept is to be extended to cover identification systems where human originate information is employed. 


\section{References}

Bastiere, A. (1997). Fusion methods for mltisensor classification of airborne targets, Aerospace Science and Technology 1: 83-94.

B.Ristic \& P.Smets (2005). Target classification approach based on the belief function theory, IEEE Transactions On Aerospace And Electronics Systems 41(2).

Carson, R. Meyer, M. \& Peters, D. (26-30 Oct 1997). Fusion of iff and radar data, 16th AIAA/IEEE Digital Avionics Systems Conference (DASC) 1: 5.3-9-15.

Deng, H. Chao, P. \& Liu, J. (2011). Entropy flow-aided navigation, The Journal of Navigation, Vol. 64, The Royal Institute of Navigation, pp. 109-125.

Dezert, J. \& Smarandache, F. (2006). Dsmt: A new paradigm shift for information fusion, Cogis ' 06 Conference, Paris .

Hall, H. \& Llinas, J. (2001). Handbook of Multisensor Data Fusion, CRC.

Harmanec, D. (1996). Uncertainty in Dempster-Shafer Theory, PhD Dissertation, State University of New York.

Hartley, R. (n.d.). Transmission of information, The Bell System Technical Journal 7(3): 535-563.

Klir, G. (2006). Uncertainty and Information, Fundations of Generalised Information theory, Wiley Interscience.

Klir, G. \& Smith, R. (2001). On measuring uncertainty and uncertainty based information: Recent developments, Annals of Mathematics and Artificial Intelligence 32: 5-33.

Leung, H. \& Wu, J. (2000). Bayesian and dempster-shafer target identification for radar surveillance, IEEE Transactions on Aerospace and Electronic Systems 36(2): 432-447.

Perlovsky, L. Chernick, J. \& Schoendorf, W. (1995). Multi-sensor atr and identification of friend or foe using mlans, Neural Networks $8(7 / 8)$.

Porretta, M. Schuster, W. \& Ochieng, W. (2010). Strategic conflict detection and resolution using aircraft intent information, The Journal of Navigation, Vol. 63, The Royal Institute of Navigation, pp. 61-88.

Schuster, W. \& Ochieng, W. (2011). Airport surface movement - critical analysis of navigation system performance requirements, The Journal of Navigation, Vol. 64, The Royal Institute of Navigation, pp. 281-294.

Shafer, G. (1976). A mathematical theory of evidence, Princeton University Press.

Yu, B. \& Sycara, K. (2006). Learning the quality of sensor data in distributed decision fusion, Proceeding of the 9th International Conference on Information Fusion. 


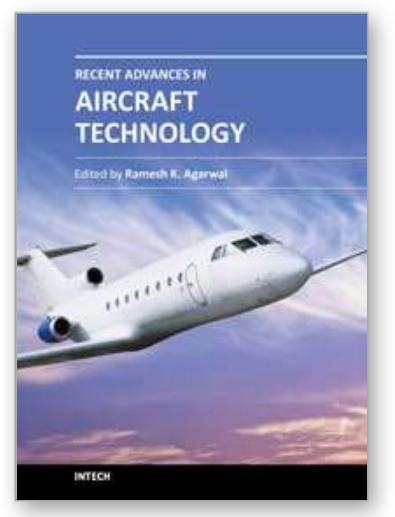

\author{
Recent Advances in Aircraft Technology \\ Edited by Dr. Ramesh Agarwal
}

ISBN 978-953-51-0150-5

Hard cover, 544 pages

Publisher InTech

Published online 24, February, 2012

Published in print edition February, 2012

The book describes the state of the art and latest advancements in technologies for various areas of aircraft systems. In particular it covers wide variety of topics in aircraft structures and advanced materials, control systems, electrical systems, inspection and maintenance, avionics and radar and some miscellaneous topics such as green aviation. The authors are leading experts in their fields. Both the researchers and the students should find the material useful in their work.

\title{
How to reference
}

In order to correctly reference this scholarly work, feel free to copy and paste the following:

Peter Pong and Subhash Challa (2012). Measuring and Managing Uncertainty Through Data Fusion for Application to Aircraft Identification System, Recent Advances in Aircraft Technology, Dr. Ramesh Agarwal (Ed.), ISBN: 978-953-51-0150-5, InTech, Available from: http://www.intechopen.com/books/recent-advancesin-aircraft-technology/measuring-and-managing-uncertainty-through-data-fusion-on-target-identification

\section{INTECH}

open science | open minds

\section{InTech Europe}

University Campus STeP Ri

Slavka Krautzeka 83/A

51000 Rijeka, Croatia

Phone: +385 (51) 770447

Fax: +385 (51) 686166

www.intechopen.com

\section{InTech China}

Unit 405, Office Block, Hotel Equatorial Shanghai

No.65, Yan An Road (West), Shanghai, 200040, China

中国上海市延安西路65号上海国际贵都大饭店办公楼 405 单元

Phone: +86-21-62489820

Fax: $+86-21-62489821$ 
(C) 2012 The Author(s). Licensee IntechOpen. This is an open access article distributed under the terms of the Creative Commons Attribution 3.0 License, which permits unrestricted use, distribution, and reproduction in any medium, provided the original work is properly cited. 